\title{
High Energy Gamma Rays
}

\author{
R. Mukherjee ${ }^{\mathrm{a}}$ \\ ${ }^{a}$ Department of Physics \& Astronomy, \\ Barnard College, Columbia University, New York, NY 10027
}

This article reviews the present status of high energy $\gamma$-ray astronomy at energies above $30 \mathrm{MeV}$. Observations in the past decade using both space- and ground-based experiments have been primarily responsible for giving a tremendous boost to our knowledge of the high energy Universe. High energy $\gamma$-rays have been detected from a wide range of Galactic and extragalactic astrophysical sources, such as $\gamma$-ray bursters, pulsars, and active galaxies. These observations have established high energy $\gamma$-ray astronomy as a vital and exciting field, that has a bright future. This review summarizes the experimental techniques, observations and results obtained with recent experiments, and concludes with a short description of future prospects.

\section{Introduction}

High energy $\gamma$-rays are one of the most direct ways of studying the non-thermal Universe. Sources of very high energy radiation enable us to explore the highest energy accelerators in the cosmos, in situations with extreme gravitational and magnetic fields. Although high energy $\gamma$-rays have been studied for some time, rapid development in the field came only in the 1990s.

The most significant thrust to high energy astronomy above $30 \mathrm{MeV}$ came with the launch of the Compton Gamma Ray Observatory (CGRO) in 1991, which carried onboard the Energetic Gamma-Ray Experiment Telescope (EGRET), along with three other instruments. Until June 2000, when CGRO was de-orbitted, EGRET produced a wealth of astrophysical results, and was responsible for the detection of more than 270 point sources of $\gamma$-rays [1]. The space-based results were complemented by ground-based atmospheric Cherenkov telescopes (ACTs), which detected $\gamma$-rays with energies above $250 \mathrm{GeV}$ from a few of the same sources. Particularly, the detection of the Crab Nebula above $250 \mathrm{GeV}$ by more than eight separate experiments has firmly established ground-based $\gamma$-ray astronomy on a solid foundation [2].

It is impossible to do justice to the exciting developments in high energy $\gamma$-ray astronomy in the few allotted pages. This article, therefore, selectively reviews a few highlights of the field, and refers the reader for more details to some of the more comprehensive reviews that have appeared in the literature recently [2]. The review is organized as follows: Sections 2 and 3 of this article summarize the techniques and results from EGRET and the ground-based experiments, respectively. While the experimental techniques are distinct, there is considerable overlap in the scientific issues addressed by the space- and groundbased experiments. The three other $\gamma$-ray experiments, BATSE, OSSE, and COMPTEL onboard CGRO are not included in this review, but are described elsewhere [6]. Some recent developments in ground-based experiments, and future directions are given in section 4 .

\section{Space-based Experiments}

\subsection{The EGRET Instrument}

EGRET was primarily a spark chamber instrument that detected $\gamma$-rays in the energy range $\sim$ $30 \mathrm{MeV}$ to $30 \mathrm{GeV}$ using the pair production technique. The instrument had a lifetime of approximately 9 years, from 1991 May to 2000 June, and proved to be immensely successful. It had the standard components of a high-energy $\gamma$-ray instrument: an anticoincidence dome to discriminate against charged particles, a spark cham- 
ber particle track detector with interspersed high$Z$ material to convert the $\gamma$-rays into electronpositron pairs, a triggering telescope to detect the presence of the pair with the correct direction of motion, and an energy measurement system, which in the case of EGRET was constructed of $\mathrm{NaI}(\mathrm{Tl})$ crystals. The anticoincidence dome was successful in rejecting the charged particle background, which outnumber the $\gamma$-rays by a factor of $10^{4}$. EGRET had an effective area of 1500 $\mathrm{cm}^{2}$ in the energy range $0.2 \mathrm{GeV}$ to $1 \mathrm{GeV}$, decreasing to about one-half the on-axis value at $18^{\circ}$ off-axis. The threshold sensitivity of EGRET $(>100 \mathrm{MeV})$ for a single 2-week observation was $\sim 3 \times 10^{-7}$ photons $\mathrm{cm}^{-2} \mathrm{~s}^{-1}$. Details of the EGRET instrument are given elsewhere [7].

Prior to EGRET, two other successful satellite experiments, SAS-2 and COS-B, pioneered the field of high energy $\gamma$-rays. These experiments were responsible for making some of the first maps of the $\gamma$-ray sky and producing the first $\gamma$-ray source catalogs [8].

\subsection{Results from EGRET \\ 2.2.1. Point Sources}

The $\gamma$-ray sky in the $30 \mathrm{MeV}$ to $30 \mathrm{GeV}$ range comprises both diffuse radiation as well as point sources. The diffuse radiation, as described in the following section, has a Galactic as well as an extragalactic component, and needs to be modeled in order to do point source analysis of the EGRET data. The point sources detected by EGRET above $100 \mathrm{MeV}$ are shown in Fig. 1. EGRET has seen several different kinds of sources, as shown in Table 1. In addition to the sources listed in the table, EGRET has also detected $5 \gamma$-ray bursts [9], the X-ray binary Cen X-3 [10], and 1 solar flare [11].

It is interesting to note that more than $60 \%$ of the EGRET sources are unidentified, with no firmly established counterparts at other wavelengths. Some of these sources have remained unidentified since the first surveys of the $\gamma$ ray sky with the COS-B satellite [8], and are one of the outstanding mysteries of the EGRET mission. Some researchers have found correlations between unidentified EGRET sources in the Galactic plane and supernova remnants 12

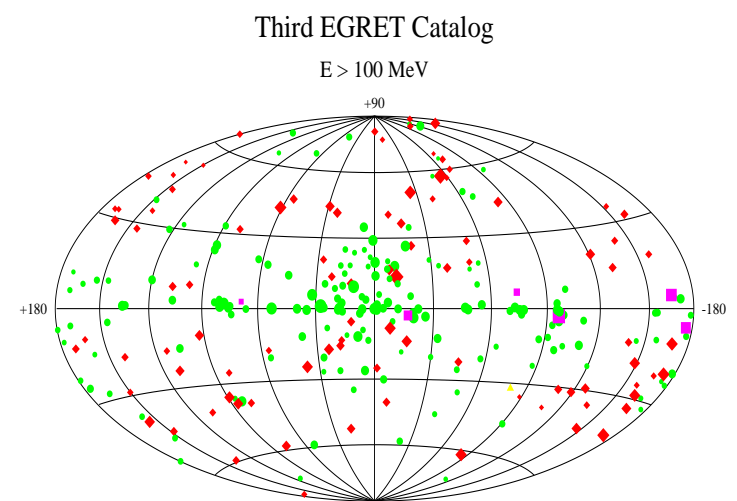

Figure 1. Point sources detected by EGRET at $>100 \mathrm{MeV}$ (Third EGRET Catalog) 1

Table 1

Sources in the Third EGRET (3EG) Catalog

\begin{tabular}{ll}
\hline Source Class & Number \\
\hline Blazars & $67(94)^{*}$ \\
Pulsars & 6 \\
Unidentified & 170 \\
Normal Galaxy & 1 (LMC) \\
Radio Galaxy & 1 (Cen A) \\
\hline
\end{tabular}

* 27 AGN have been marginally identified.

14], while others report correlations with $\mathrm{OB}$ associations and massive stars 15, 16. A review of the various efforts to understand the nature of these sources is given in 17]. Recently, it has been noted that the mid-latitude unidentified EGRET sources form a population distinctly different from the sources along the Galactic plane [18, 19. Further, it has been suggested that these mid-latitude sources are probably associated with the Gould Belt of massive stars and gas clouds, about 600 light years away [18]. Counterparts to individual unidentified sources may only be found after the launch of future, more sensitive $\gamma$-ray missions such as GLAST (see section 4).

The majority of the sources away from the Galactic plane are extragalactic and have been identified with active galactic nuclei (AGN). Other than the unidentified sources, AGN consti- 
tute the largest class of EGRET sources. EGRET tends to detect the "blazar" class of AGN, which are characterized by emissions that include high radio and optical polarization, and rapid flux variability at all wavelengths. A large fraction of these sources exhibit apparent superluminal motion, as evidenced from VLBI radio observations. EGRET has observed variability in several blazars on timescales of days to months. A review on the blazars observed by EGRET may be found in [20].

The $\gamma$-ray spectrum of blazars in the $30 \mathrm{MeV}$ to $30 \mathrm{GeV}$ range is well-described by a single power-law of spectral index $\sim 2.0$. The broad-band spectral energy distribution (SED) of one EGRET flat-spectrum radio quasar (FSRQ), PKS 0528+134, is shown in Fig. 2. The figure demonstrates the typical characteristics of the SED of a quasar observed by EGRET, showing that the $\gamma$-ray luminosity dominates the spectrum. In fact, in most FSRQs the $\gamma$-ray power dominates or equals the power at other wavebands. The apparent luminosity, assuming isotropic emission, is $10^{48}-10^{50} \mathrm{ergs} \mathrm{s}^{-1}$ [22]. Fig. 2 includes broadband observations at several different epochs and demonstrates another important characteristic of EGRET blazars - spectral variability between different states of the source.

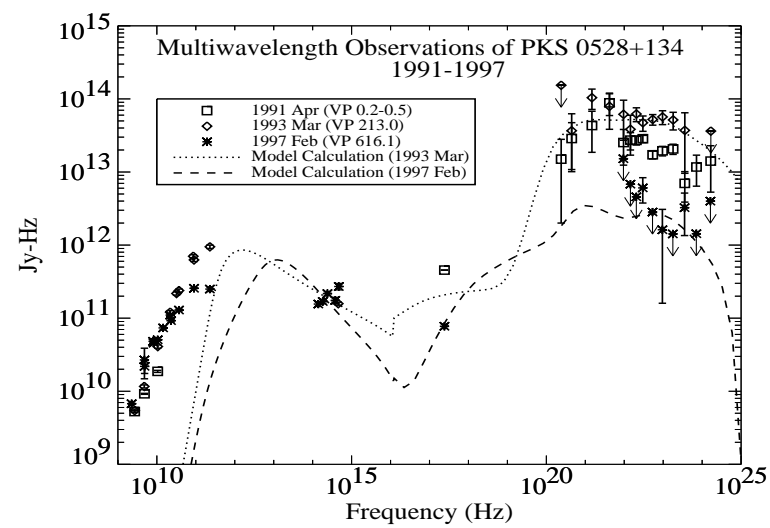

Figure 2. SEDs of the blazar PKS 0528+134 during several different epochs [21]
EGRET observations of blazars help to constrain models of emission for these objects. Rapid variability, high compactness, and superluminal motion suggest that the emission is likely to be beamed and Doppler-boosted into the line of sight [23]. Simplifying many details, it is generally believed that AGN are powered by accretion of matter on to a supermassive black hole. For the case of blazars, collimated jets composed of shocks or plasma blobs move with relativistic speeds outward along the axes of the accretion disk, making a small angle with the axis.

Gamma-ray emission models for blazars are generally divided into two broad classes: leptonic and hadronic. Details of these models are not discussed here but may be found in [20]. Fig. 2 shows an example of a leptonic jet model applied to the case of PKS 0528+134 [21]. It has been suggested that hadronic models may be distinguished from leptonic models by the observation of high energy neutrinos that may be produced as a result of photoproduction [24]. There are several outstanding questions regarding the details of the different models that may only be resolved in the future.

In addition to blazars, EGRET has detected $\gamma$-rays from 6 pulsars. These are the Crab, Vela, PSR 1706-44, B1951+31, B1055-58, and Geminga. In addition, soft $\gamma$-rays have been seen from the pulsar B1509-58, although no pulse was detected in the EGRET energy range. A few other pulsars have been marginally identified. All strongly-detected $\gamma$-ray pulsars are doublepulsed, although the light curves exhibit a wide variety of patterns. Unlike blazars, the flux history of pulsars show relatively constant emission over time. The $\gamma$-ray spectra of these sources have power-law indices in the range -1.39 to 2.07. Geminga is an interesting pulsar, as it is radio-quiet, and its period was originally derived from X-ray measurements. An up-to-date review on EGRET pulsars and how they impact models of pulsar $\gamma$-ray emission may be found in 25.

One of the most exciting results in high energy astrophysics is undoubtedly the detection of $\gamma$ ray bursts (GRB). More than 2500 bursts have been recorded by BATSE, but the detection of 5 of these bursts by EGRET has demonstrated 
that GRB are not necessarily a low-energy $\gamma$-ray phenomena. A review on the high energy emission from GRB is presented elsewhere in these proceedings 26. EGRET-detected bursts are described in [9].

\subsubsection{Diffuse Radiation}

EGRET observations of the $\gamma$-ray sky above $30 \mathrm{MeV}$ have provided a unique view of the diffuse $\gamma$-ray radiation, which constitutes the bulk of the emission detected by EGRET. The diffuse emission is found to have a Galactic component arising from cosmic-ray interactions with the local interstellar gas and radiation [27], as well as an almost uniformly distributed component believed to originate outside the Galaxy 28. The average spectrum of the extragalactic diffuse emission is well-described by a single power-law with an index $-(2.10 \pm 0.03)$ in the $30 \mathrm{MeV}$ to $100 \mathrm{GeV}$ range.

The precise origin of the extragalactic diffuse emission is not well-known, and possibly includes both diffuse origin as well as contributions from unresolved point sources. These are discussed in 28]. Recent theories include contributions by the upscattering of cosmic microwave background radiation by highly relativistic electrons, formed as a result of gravity-induced shock waves during the formation of large scale structure in the intergalactic medium 29]. Models based on discrete source contributions have considered an array of different source classes. Of these the most popular have been unresolved blazars. Recent estimates of blazar contribution based on a $\gamma$-ray evolution function derived from $\gamma$-ray data is roughly 25 to 30 percent [30], although earlier estimations have found a larger contribution [31].

\section{Ground-based Experiments}

\subsection{Instruments $(>300 \mathrm{GeV})$}

At very high energies satellite experiments are made impractical due to the rapidly falling photon fluxes. However, at these energies $\gamma$-ray astronomy can be done using ground-based instruments by detecting the secondary particles or radiation produced as a result of the interaction of high energy $\gamma$-rays in the upper atmosphere.
At energies > $300 \mathrm{GeV}$, imaging atmospheric Cherenkov telescopes (ACTs) have been used successfully to image the flashes of Cherenkov light produced as a result of the electromagnetic cascades initiated by high energy $\gamma$-rays. The field was pioneered by the Whipple 10-m telescope, which was the first to detect $\mathrm{TeV} \gamma$-rays from an astrophysical source 32]. Imaging ACTs detect the Cherenkov flashes using fast photomultipliers (PMTs) located at the focal plane of large optical reflectors. The PMT signals are subsequently read out using a fast electronics chain. In comparison to satellite experiments, ground-based ACTs have much larger collection areas, and better angular resolution, although much smaller fields of view. ACTs can only operate on cloudless, moonless nights, and thus have a lower duty cycle.

The Whipple Observatory was followed by several successful high resolution ACTs, e.g., CAT, CANGAROO, Durham, GT-48, HEGRA, Pachmari, SHALON, TACTIC, Telescope Array, among others. A complete list of current telescopes and their properties may be found in [3.5]. Air shower arrays, which operate above $30 \mathrm{TeV}$, and directly detect the secondary particles in the air showers are not included in the review (see [5] for a discussion).

\subsection{Results}

Figure 3 shows the skymap of point sources detected by ground-based experiments at energies $>250 \mathrm{GeV}$. In addition to those shown in the figure, a few other sources have been detected at a lower significance 33. The source list consists of both Galactic, as well extragalactic objects.

The Crab Nebula was the first source to be detected unambiguously in $\mathrm{TeV} \gamma$-rays. Since then it has been detected several times with various different instruments and is clearly the standard candle in the $300 \mathrm{GeV}$ to $3 \mathrm{TeV}$ range [33]. No pulsed emission has been observed from the Crab at $\mathrm{TeV}$ energies. In addition to the Crab, two other plerionic supernova remnants (SNRs), Vela and PSR 1706-44, and a couple of shell-type SNRs have been detected by ACTs (see [2,33] for a review).

Mrk 421 was the first extragalactic source to be detected at $>250 \mathrm{GeV}$ 34, and has been ob- 


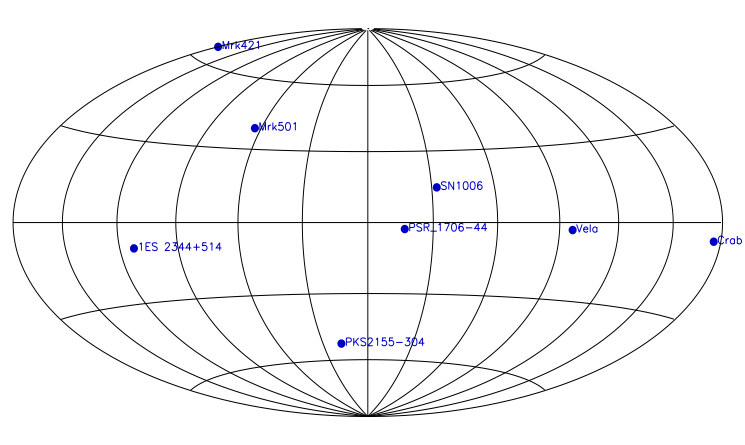

Figure 3. Locations of the $\gamma$-ray point sources in Galactic coordinates detected by ground-based ACTs $(250 \mathrm{GeV}$ to $15 \mathrm{TeV})$.

served by several different ground-based instruments [2]. At a redshift of 0.031 , it is the closest BL Lac object seen by EGRET. The source exhibits extremely rapid variability at $\mathrm{TeV}$ energies with doubling times of the order of 1 hour or less 35. Its $\mathrm{TeV}$ flux can vary from a quiescent level of 0.3 to $10 \times$ Crab flux during a $\gamma$-ray flare. Mrk 421 exhibits the fastest time scale $\gamma$-ray variability seen in any blazar to date. Figure 4 shows the spectacular flare from the source in 1996. Crosscorrelations of various data sets of Mrk 421 indicate a significant correlation of the X-ray and $\mathrm{TeV} \gamma$-ray flux variability [36].

At a redshift of 0.033 , Mrk 501 is the second closest X-ray-selected BL Lac (XBL) known and was the second blazar discovered at $\mathrm{TeV}$ energies by the Whipple group [37]. It has since been observed by HEGRA, CAT, TACTIC, and the Telescope Array Project 38 41. The detection of Mrk 501 in the EGRET data came only after the $\mathrm{TeV}$ detection [42], making it the first object to be discovered as a $\gamma$-ray source from the ground. The broadband spectrum of Mrk 501 during the outburst of 1996 May is shown in Fig. 5 [42]. EGRET reported a $\mathrm{GeV}$ outburst from the source when Mrk 501 was detected at $5.3 \sigma$ above 500 $\mathrm{GeV}$ [43. Mrk 501 also shows significant variability at $\mathrm{X}$-ray, low-energy $\gamma$-ray and $\mathrm{TeV}$ energies. This source was detected for the first time in the $100 \mathrm{keV}$ to $1 \mathrm{MeV}$ range by OSSE, the result-

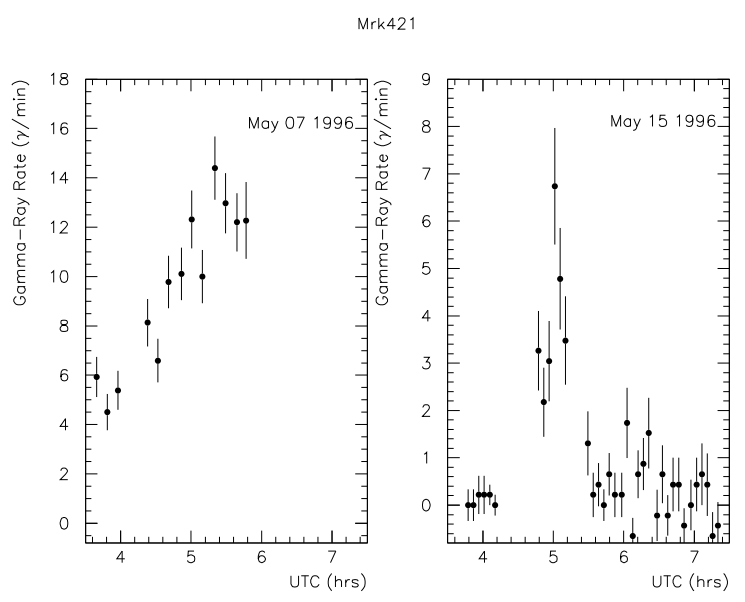

Figure 4. Flux of TeV photons from Mrk 421 as a function of time for two separate flares, indicating variability on the time scale $<$ few hours [35]

ing SED showing this emission to be most likely of synchrotron origin [44]. This represents the highest synchrotron cutoff seen in any blazar at present. At $\mathrm{TeV}$ energies, the source exhibited an extraordinary outburst in 1997 [45], when its intensity was observed to be several times that of the Crab.

A comprehensive review of the other sources detected at $\mathrm{TeV}$ energies is given in [2]. A comparison of the $\mathrm{GeV}$ and $\mathrm{TeV}$ skymaps show that only a handful of the EGRET sources have been detected at $\mathrm{TeV}$ energies, although a simple extrapolation of the EGRET spectra predict flux levels well above the sensitivity of current atmospheric Cherenkov telescopes. The cutoffs in the spectra could be either due to intergalactic absorption or intrinsic absorption at the source. $\gamma$-rays traversing intergalactic distances may be absorbed by photon-photon pair production $\left(\gamma \gamma \rightarrow e^{+} e^{-}\right)$, with a $\mathrm{TeV}$ photon preferentially interacting with $0.5 \mathrm{eV}$ (IR) background photon. The presence of this extragalactic background light (EBL) is probably the main explanation for the lack of detection of the majority of EGRET sources at $\mathrm{TeV}$ energies. The spectral cutoffs of the sources is expected to be in the 50 to $250 \mathrm{GeV}$ range, and is one of the primary motivations for exploring this regime. The study of the 


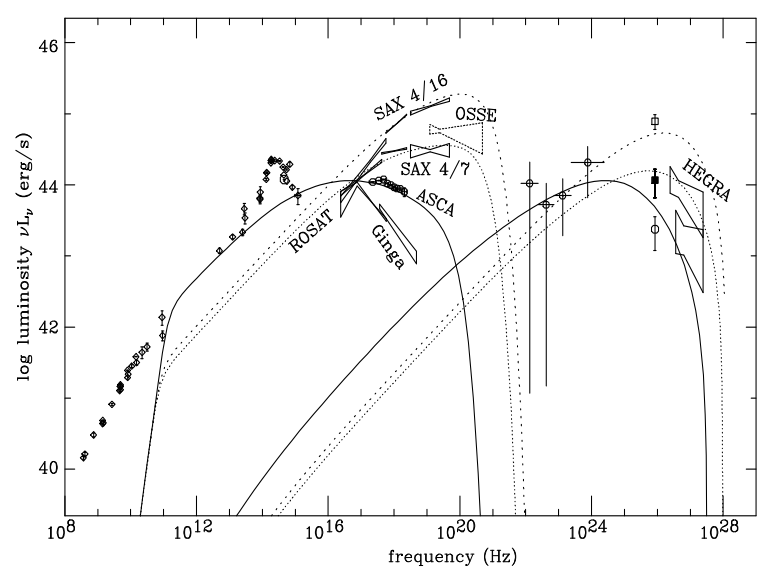

Figure 5. Broadband SED of Mrk 501 during several different epochs. The filled and open squares correspond to Whipple flux on 1997 April 7 and 16, respectively. The circles correspond to data taken in 1996 March. The diamonds correspond to non-simultaneous data from the NED data base. 42

EBL is important for several reasons. Direct measurements of the EBL are sparsely sampled and fraught with uncertainties due to the presence of foreground IR radiation 46, 47]. The TeV spectra of Mrk 421 and Mrk 501 have been used to indirectly derive limits on the EBL 48,49 . Clearly, more spectral measurements of AGN at different redshifts, particularly in the 50 to $250 \mathrm{GeV}$ range, are required in order to set restrictive limits on the EBL.

\section{The Future of High Energy Gamma-ray Astronomy}

The future prospects of high energy astronomy are very promising. Recent results from spaceand ground-based experiments have raised numerous important questions about the emission mechanisms in astrophysical sources. There is also the tantalizing possibility of probing novel astrophysical phenomena which could arise as a result of new physics beyond the Standard Model
501. In the future, it is clear that one needs more sensitive instruments, both satellite-borne as well as ground-based. It is particularly important to explore the energy region between 20 and $250 \mathrm{GeV}$.

Recently, two experiments STACEE and CELESTE have demonstrated that lower energy thresholds can be achieved by using existing large arrays of solar heliostat mirrors to collect Cherenkov light. Both these experiments have reported the detection of the Crab nebula 51,52 and are in the final stages of construction. A third experiment, Solar-2 is currently being built [53]. For the first time, the energy gap between space- and ground-based experiments is starting to be bridged. Experiments that will be built in the future include MAGIC in Spain, HESS in Namibia, VERITAS in Arizona, and SuperCANGAROO in Australia (see [2] and references therein). The project GRACE in India will involve four independent experimental systems that will span nearly ten decades of photon energy $(\sim 10 \mathrm{keV}-100 \mathrm{TeV})$ and do coordinated studies of $\gamma$-ray sources [54]. These experiments will complement each other and together span the energy range from $20 \mathrm{GeV}$ to $10 \mathrm{TeV}$. In addition to the ACTs, MILAGRO, a new kind of $\gamma$-ray detector with a large field-of-view and continuous operation has recently come on line. A prototype of this detector, Milagrito, reported the possible detection of the correlated $\mathrm{TeV} \gamma$-ray burst GRB 970417a [56], as well as Mrk 501 [57].

The ground-based experiments will be complemented by two next generation space experiments, GLAST and AGILE. GLAST, which is expected to be launched in 2005, is projected to be a state-of-the art detector which will use a Sistrip tracker and a CsI calorimeter 58]. GLAST's sensitivity will be a factor of $>30$ better than EGRET, and is expected to detect two orders of magnitude more sources than EGRET. GLAST will have some overlap with ground-based instruments in the 30-300 GeV regime. A smaller EGRET-sized experiment, the Italian AGILE [59], sensitive in $30 \mathrm{MeV}$ to $50 \mathrm{GeV}$ energy range, is expected to be launched in 2002 and bridge the gap before GLAST. Experiments in high energy $\gamma$-rays will be complemented by neutrino and cos- 
mic ray experiments [1], thus promising significant scientific progress in the future.

I wish to thank M. Catanese, J. Kataoka, R. Ong, and P. Sreekumar for providing some of the data and figures presented here, as well as at the Neutrino 2000 conference, and D. Hanna and B. Dingus for reading the manuscript. This research is supported in part by the National Science Foundation and by an award from Research Corporation.

\section{REFERENCES}

1. R. C. Hartman, et al., ApJ, 123 (1999) 79.

2. M. Catanese \& T. C. weekes, astro-ph 9906501 (1999).

3. R. A. Ong, Phys. Rep., 305 (1998) 95.

4. R. A. Ong, astro-ph 0003014 (2000).

5. C. M. Hoffman, et al., Rev. Mod. Phys., 71 (1999) 897.

6. N. Gehrels \& C. Shrader, AIP Conf. Proc. 410 (1997) 3.

7. D. J. Thompson, et al., ApJS, 102 (1995) 259.

8. B. N. Swanenburg, et al., ApJ, 243 (1981) L69.

9. B. L. Dingus, J. R. Catelli, E. J. Schneid, AIP Conf. Proc. 428 (1998) 349.

10. W. T. Vestrand, P. Sreekumar, \& M. Mori, ApJ, 483 (1997) L49.

11. G. Kanbach, et al., A\&AS, 97 (1993) 349.

12. I.-A. Yadigaroglu \& R. W. Romani, ApJ, 476 (1997) 356.

13. S. J. Sturner \& C. D. Dermer, A\&A, 281 (1995) L17.

14. J. A. Esposito, et al., ApJ, 461 (1996) 820.

15. P. Kaaret \& J. Cottam, ApJ, 462 (1998) L65.

16. G. E. Romero, et al., A\&A, 348 (1999) 868.

17. R. Mukherjee, I. A. Grenier, D. J. Thompson, AIP Conf. Proc. 410 (1997) 384.

18. N. Gehrels, et al., Nature, 404 (2000) 363.

19. I. A. Grenier, Nature, 404 (2000) 344.

20. R. C. Hartman, et al., AIP Conf. Proc. 410 (1997) 307.

21. R. Mukherjee, et al., ApJ, 527 (1999) 132.

22. R. Mukherjee, et al., ApJ, 490 (1997) 116.

23. R. Schlickeiser, Sp. Sci. Rev., 75 (1996) 299.

24. A. Muecke, astro-ph 0004052

25. D. J. Thompson, $\gamma 2000$ Proc., Heidelberg.
26. E. Waxman, Neutrino 2000 Proc., Sudbury.

27. S. D. Hunter, et al., ApJ, 481 (1997) 205.

28. P. Sreekumar, et al., ApJ, 494 (1998) 523.

29. A. Loeb \& E. Waxman, Nature, 405 (2000) 156.

30. J. Chiang \& R. Mukherjee, ApJ, 496 (1998) 752 .

31. F. W. Stecker \& M. H. Salamon, ApJ, 464 (1996) 600.

32. T. C. Weekes, et al., ApJ, 342 (1989) 379.

33. T. C. Weekes, et al., astro-ph/9910394.

34. M. Punch, et al., Nature, 358 (1992) 477.

35. J. Gaidos, et al., Nature, 383 (1996) 319.

36. J. H. Buckley, et al., ApJ, 472 (1996) L9.

37. J. Quinn, et al., ApJ, 456 (1996) L83.

38. S. M. Bradbury, et al., A\&A, 320 (1997) L5.

39. M. Punch, et al., 25th ICRC, 3 (1997) 253.

40. C. L. Bhat, IAUC (1997) 6709.

41. N. Hayashida, et al., ApJ, 504 (1998) L71.

42. J. Kataoka, et al., ApJ, 514 (1999) 138.

43. P. Sreekumar, et al., AIP Conf. Proc. 510 (1999) 318.

44. M. Catanese, et al., ApJ, 487 (1997) L143.

45. F. Aharonian, et al., A\&A, 349 (1999) 11.

46. M. G. Hauser, et al., ApJ, 508 (1998) 25.

47. J.-L. Puget, et al., A\&A, 308 (1996) L5.

48. S. D. Biller, et al., Phys. Rev. Lett., 80 (1998) 2992.

49. T. Stanev \& A. Franceschini, ApJ, 494 (1998), L59.

50. G. Jungman, M. Kamionkowski, \& K. Griest, Phys. Rep., 267 (1996) 195.

51. S. Oser, et al., 26th ICRC, 3 (1999) 464.

52. M. de Naurois, 26th ICRC, 5 (1999) 211.

53. T. Tumer, et al., APh, 11 (1999) 271.

54. C. L. Bhat, "Towards a Major Atmospheric Cerenkov Detector-V," Kruger Park, South Africa (1997).

55. G. Sinnis, et al., Nucl. Phys. B (Proc. Suppl.), 43 (1995) 141.

56. R. Atkins, et al., ApJ, 533 (2000) L119.

57. R. Atkins, et al., ApJ, 525 (1999) L25.

58. N. Gehrels \& P. Michelson, APh, 526 (1999) 297.

59. M. Tavani, et al., A\&AS, 138 (1999) 569. 